\title{
COMPLETION OF NORMED LINEAR SPACES
}

\section{KUNG-WEI YANG}

1. Introduction. In this paper, we show that the completion functor from the category of normed linear spaces and contractions to the category of Banach spaces and contractions is "normal exact." An application of the main theorem is also included.

2. Notation and definitions. Let $F$ be the field of real numbers or the field of complex numbers. The completion $\Lambda(X)$ of a normed $F$-linear space $X$ is usually constructed as the quotient $\Lambda(X)$ $=C(X) / \operatorname{co}(X)$ of the normed linear space $C(X)$ of all Cauchy sequences $\left[x_{1}, x_{2}, \cdots\right]$ in $X$ (with norm $\left|\left[x_{1}, x_{2}, \cdots\right]\right|=\sup _{i}\left|x_{i}\right|$ ) by the subspace $\operatorname{co}(X)$ of sequences tending to zero.

It is well known that the passage $X \mapsto \Lambda(X)$ is a functor from the category $N^{*}$ of normed linear spaces and contractions (continuous linear maps $f: X \rightarrow Y$ such that $|f| \leqq 1$ ) to the full subcategory $B^{\bullet}$ of $N^{*}$ consisting of Banach spaces. Indeed, $\Lambda$ is just the (left) adjoint to the inclusion of $B^{*}$ in $N^{*}$, and the front adjunction $c(X): X \rightarrow \Lambda(X)$ is just the composition $c(X)=m(X) l(X)$, where $l(X): X \rightarrow C(X)$ is defined by $l(X)(x)=[x, x, \cdots]$ and $m(X)$ is the quotient projection $C(X) \rightarrow \mathbf{\Lambda}(X)$.

It is useful to note that $X \mapsto C(X)$ and $X \mapsto \operatorname{co}(X)$ are functors from $N^{*}$ to $N^{*}$ (if $f: X \rightarrow Y$, then $C(f): C(X) \rightarrow C(Y)$ is the map $\left.C(f)\left(\left[x_{1}, x_{2}, \cdots\right]\right)=\left[f\left(x_{1}\right), f\left(x_{2}\right), \cdots\right]\right)$ and co is a subfunctor of $C$. We will denote by $n:$ co $\rightarrow C$ the inclusion. Note also that $l$ defined above is a natural transformation from the identity functor on $N^{\bullet}$ to the functor $C$ and $m$ is a natural transformation from the functor $C$ to the functor $\Lambda$.

Definition. A (finite or infinite) sequence in $N^{*}$ (or $B^{*}$ )

$$
\cdots \rightarrow X_{n-1} \stackrel{f_{n-1}}{\longrightarrow} X_{n} \stackrel{f_{n}}{\longrightarrow} X_{n+1} \rightarrow \cdots
$$

is normal exact, if

(i) for each $n, f_{n}^{-1}(0)=f_{n-1}\left(X_{n-1}\right)$, and

(ii) for each $n$,

$$
X_{n} / f_{n}^{-1}(0) \stackrel{f_{n}^{\prime}}{\longrightarrow} f_{n}\left(X_{n}\right),
$$

where $f_{n}^{\prime}$ is the map induced by $f_{n}$, is an isometry.

It is easy to see that for each $X$ in $N^{\cdot}$ the sequences

Received by the editors March 23, 1967. 


$$
0 \rightarrow X \stackrel{c(X)}{\longrightarrow} \Lambda(X) \text { and } 0 \rightarrow \operatorname{co}(X) \stackrel{n(X)}{\longrightarrow} C(X) \stackrel{m(X)}{\longrightarrow} \Lambda(X) \rightarrow 0
$$

are normal exact.

\section{The main result.}

TheOREM. If

$$
0 \rightarrow Y \stackrel{i}{\rightarrow} X \stackrel{p}{\rightarrow} Z \rightarrow 0
$$

is a normal exact sequence in $N^{*}$, then

$$
0 \rightarrow \Lambda(Y) \stackrel{\Lambda(i)}{\longrightarrow} \Lambda(X) \stackrel{\Lambda(p)}{\longrightarrow} \Lambda(Z) \rightarrow 0
$$

is a normal exact sequence in $B^{\circ}$. (Therefore, we can say that the completion functor $\Lambda$ is "normal exact.")

Lemma 1. If

$$
0 \rightarrow Y \stackrel{i}{\rightarrow} X \stackrel{p}{\rightarrow} Z \rightarrow 0
$$

is a normal exact sequence in $N^{*}$, then

$$
0 \rightarrow \operatorname{co}(Y) \stackrel{\operatorname{co}(i)}{\longrightarrow} \operatorname{co}(X) \stackrel{\operatorname{co}(p)}{\longrightarrow} \operatorname{co}(Z) \rightarrow 0
$$

is a normal exact sequence in $N$.

Proof. There are only two things that need to be verified:

$$
\operatorname{co}(X) \stackrel{\operatorname{co}(p)}{\longrightarrow} \operatorname{co}(Z)
$$

is onto, and

$$
\operatorname{co}(X) / \operatorname{co}(i)(\operatorname{co}(Y)) \stackrel{\operatorname{co}(p)^{\prime}}{\longrightarrow} \operatorname{co}(Z),
$$

where $\operatorname{co}(p)^{\prime}$ is the map induced by $\operatorname{co}(p)$, is an isometry. Everything else is obvious. To prove (1), we let $\left[z_{1}, z_{2}, \cdots\right] \in \operatorname{co}(Z)$. Since

$$
0 \rightarrow Y \stackrel{i}{\rightarrow} X \stackrel{p}{\rightarrow} Z \rightarrow 0
$$

is a normal exact sequence, for each $z_{k}(k=1,2, \cdots)$, there exists $x_{k} \in X$ such that $p\left(x_{k}\right)=z_{k}$ and $\left|x_{k}\right|<\left|z_{k}\right|+1 / k$. Clearly, $\left[x_{1}, x_{2}, \cdots\right]$ $\in \operatorname{co}(X)$ and $\operatorname{co}(p)\left(\left[x_{1}, x_{2}, \cdots\right]\right)=\left[z_{1}, z_{2}, \cdots\right]$. To prove (2), we first recall the definition of norms in $\operatorname{co}(X) / \operatorname{co}(i)(\operatorname{co}(Y))$ and in $\operatorname{co}(Z)$. Let $j: \operatorname{co}(X) \rightarrow \operatorname{co}(X) / \operatorname{co}(i)(\operatorname{co}(Y))$ be the canonical map. Let $\xi \in \operatorname{co}(X) / \operatorname{co}(i)(\operatorname{co}(Y))$. Then 


$$
|\xi|=\inf _{\mathcal{J}\left(\left[x_{1}, x_{2}, \cdots\right]\right)=\xi}\left\{\left|\left[x_{1}, x_{2}, \cdots\right]\right|\right\}=\inf _{\mathcal{J}\left(\left[x_{1}, x_{2}, \cdots\right]\right)=\xi}\left\{\sup _{n}\left\{\left|x_{n}\right|\right\}\right\} .
$$

On the other hand, if $\left[z_{1}, z_{2}, \cdots\right] \in \operatorname{co}(Z)$, then

$$
\left|\left[z_{1}, z_{2}, \cdots\right]\right|=\sup _{n}\left\{\left|z_{n}\right|\right\}=\sup _{n}\left\{\inf _{p\left(x_{n}\right)=z_{n}}\left\{\left|x_{n}\right|\right\}\right\} .
$$

Now, if $\xi \in \operatorname{co}(X) / \operatorname{co}(i)(\operatorname{co}(Y))$, then $\operatorname{co}(p)^{\prime}(\xi)=\left[p\left(x_{1}\right), p\left(x_{2}\right), \cdots\right]$, where $\left[x_{1}, x_{2}, \cdots\right]$ is any element satisfying $j\left(\left[x_{1}, x_{2}, \cdots\right]\right)=\xi$. Comparing the above two norms, it is clear that $\operatorname{co}(p)^{\prime}$ is an isometry.

LEMMA 2. If

$$
0 \rightarrow Y \stackrel{i}{\rightarrow} X \stackrel{p}{\rightarrow} Z \rightarrow 0
$$

is a normal exact sequence in $N^{*}$, then

$$
0 \rightarrow C(Y) \stackrel{C(i)}{\longrightarrow} C(X) \stackrel{C(p)}{\longrightarrow} C(Z) \rightarrow 0
$$

is a normal exact sequence in $N^{*}$.

Proof. As in Lemma 1, we only need to verify the following two statements:

$$
C(X) \stackrel{C(p)}{\longrightarrow} C(Z)
$$

is onto, and

$$
C(X) / C(i)(C(Y)) \stackrel{C(p)^{\prime}}{\longrightarrow} C(Z)
$$

is an isometry (where $C(p)^{\prime}$ is the map induced by $C(p)$ ). To prove (1), we let $\left[z_{1}, z_{2}, \cdots\right] \in C(Z)$. Then, for each integer $k$, there exists a positive integer $q(k)$ such that if $m(1)$ and $m(2)$ are $>q(k)$, then $\left|z_{m(1)}-z_{m(2)}\right|<\frac{1}{2} k+2$. We shall choose $q(k)$ so that the sequence $q(1)$, $q(2), \cdots$ is strictly increasing. Since

$$
0 \rightarrow Y \stackrel{i}{\rightarrow} X \stackrel{p}{\rightarrow} Z \rightarrow 0
$$

is a normal exact sequence, for $k \geqq 2$, there exists some $u_{q(k)} \in X$ such that $p\left(u_{q(k)}\right)=z_{q(k)}-z_{q(k-1)}$ and $\left|u_{q(k)}\right|<\left|z_{q(k)}-z_{q(k-1)}\right|+\frac{1}{2} k+2<\frac{1}{2} k+1$. For the same reason, if $q(k) \leqq n<q(k+1)$, and if $n=q(k)+m$, then there exists an element $t_{q(k), m} \in X$ such that $p\left(t_{q(k), m}\right)=z_{n}-z_{q(k)}$ and $\left|t_{q(k), m}\right|<\left|z_{n}-z_{q(k)}\right|+\frac{1}{2}^{k+2}<\frac{1}{2}{ }^{k+1}\left(t_{q(k), 0}\right.$ can be and shall be chosen to be equal to 0$)$. Now, let $x_{q(1)}$ be an arbitrary element such that 
$p\left(x_{q(1)}\right)=z_{q(1)}$ and for $k \geqq 2$, let

$$
s_{a(k)}=x_{q(1)}+u_{q(2)}+\cdots+u_{q_{(k)}}\left(s_{q_{(1)}}=x_{q(1)}\right) .
$$

Finally let

$$
\begin{aligned}
x_{n} & =\text { any element in } X \text { such that } p\left(x_{n}\right)=z_{n}, \text { if } n=1,2, \cdots, q(1)-1, \\
& =s_{q(k)}+t_{q(k), m}, \text { if } q(k) \leqq n<q(k+1) \text { and } n=q(k)+m .
\end{aligned}
$$

Then, $p\left(x_{n}\right)=z_{n}$, and for any $\epsilon>0$, there exists a positive integer $M$ such that $\left|x_{m}-x_{n}\right|<\epsilon$, if $m, n>M$. This means $\left[x_{1}, x_{2}, \cdots\right] \in C(X)$ and $C(p)\left(\left[x_{1}, x_{2}, \cdots\right]\right)=\left[z_{1}, z_{2}, \cdots\right]$. The proof of part (2) is similar to that of part (2) of Lemma 1.

Proof of the Theorem. All we have to do is to show that the sequence $\left({ }^{*}\right)$ enjoys conditions (i) and (ii) of the definition of normal exactness.

First, we shall prove that condition (i) is satisfied. By Lemma 1 and Lemma 2, we have the following commutative diagram:

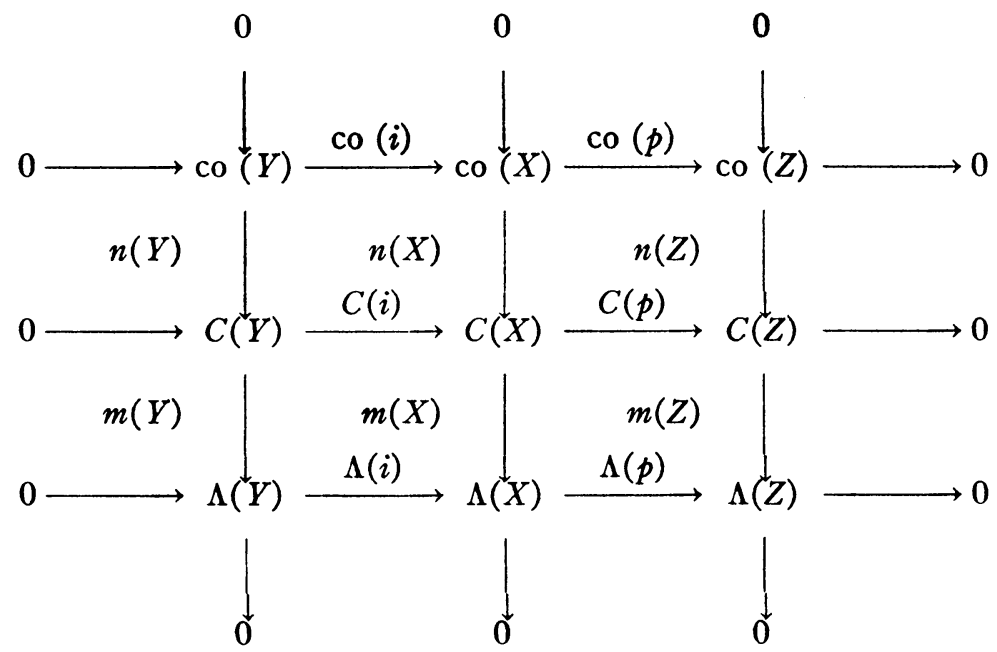

where all the columns and rows, except the last row, satisfy condition (i). By the usual diagram-chasing, it is easy to see that the sequence (*) enjoys condition (i).

To prove that the sequence $\left({ }^{*}\right)$ enjoys condition (ii), we have to show that:

(1) $\Lambda(i)^{\prime}: \Lambda(Y) \rightarrow \Lambda(i)(\Lambda(Y))$ is an isometry (where $\Lambda(i)^{\prime}$ is the map induced by $\Lambda(i))$, and

(2) $\Lambda(p)^{\prime}: \Lambda(X) / \Lambda(i)(\Lambda(Y)) \rightarrow \Lambda(Z)$ is an isometry (where $\Lambda(p)^{\prime}$ is the map induced by $\Lambda(p))$. To prove (1), let $\eta \in \Lambda(Y)$ and let 
$\left[y_{1}, y_{2}, \ldots\right] \in C(Y)$ such that $m(Y)\left(\left[y_{1}, y_{2}, \cdots\right]\right)=\eta$. We observe that $|\eta|=\lim _{k \rightarrow \infty}\left|y_{k}\right|$. Similarly, if $\xi \in \Lambda(X)$ and $\left[x_{1}, x_{2}, \cdots\right] \in C(X)$ such that $m(X)\left(\left[x_{1}, x_{2}, \ldots\right]\right)=\xi$, then $|\xi|=\lim _{k \rightarrow \infty}\left|x_{k}\right|$. From these observations, it is clear that $\Lambda(i)^{\prime}$ is an isometry. To prove (2), we first recall the definition of norms in $\Lambda(X) / \Lambda(i)(\Lambda(Y))$ and in $\Lambda(Z)$. Let $J: \Lambda(X) \rightarrow \Lambda(X) / \Lambda(i)(\Lambda(Y))$ be the canonical map. Let $A$ $\in \Lambda(X) / \Lambda(i)(\Lambda(Y))$. Then

$$
|A|=\inf _{J(\xi)=A}\{|\xi|\}=\inf _{J(\xi)=A}\left\{\lim _{k \rightarrow \infty}\left|x_{k}\right|\right\}
$$

where $m(X)\left(\left[x_{1}, x_{2}, \cdots\right]\right)=\xi$. On the other hand, if $\alpha \in \Lambda(Z)$, and if $m(Z)\left(\left[z_{1}, z_{2}, \cdots\right]\right)=\alpha$, then

$$
|\alpha|=\lim _{k \rightarrow \infty}\left|z_{k}\right|=\lim _{k \rightarrow \infty} \inf _{p\left(x_{k}\right)=z_{k}}\left\{\left|x_{k}\right|\right\} .
$$

We know that if $A \in \Lambda(X) / \Lambda(i)(\Lambda(Y))$, then $\Lambda(p)^{\prime}(A)=\alpha$, where $\alpha$ satisfied $\Lambda(p)(\xi)=\alpha$ and $\xi$ is any element such that $A=J(\xi)$. It is now clear that $\Lambda(p)^{\prime}$ is indeed an isometry. This completes the proof of the theorem.

\section{An application.}

Proposition. Let

$$
0 \rightarrow Y \stackrel{i}{\rightarrow} X \stackrel{p}{\rightarrow} X \rightarrow Z \rightarrow 0
$$

be a normal exact sequence in $N^{*}$. Then $X$ is complete $\Leftrightarrow Y$ and $Z$ are.

Proof. By the Theorem, we have the following commutative diagram:

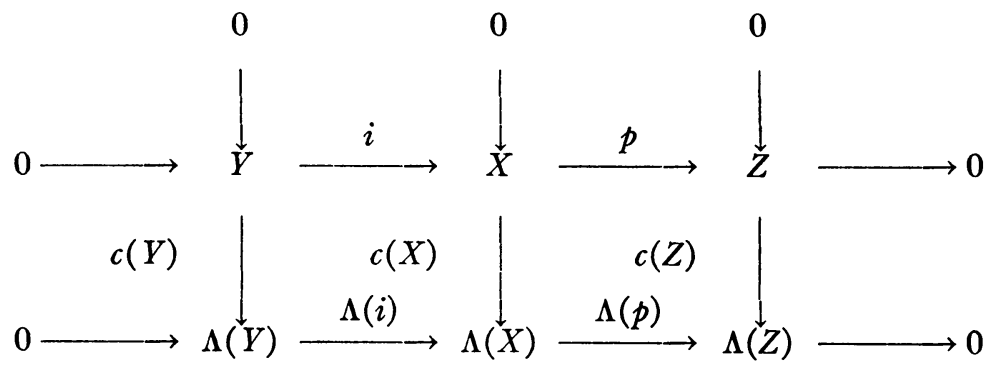

where all the rows and columns are normal exact. Let $L$ be the category of $F$-linear spaces and $F$-linear maps. Let $V: N^{*} \rightarrow L$ be the forgetful functor. Clearly, $V$ carries normal exact sequences into exact 
sequences. Applying $V$ to the above diagram and using "9-Lemma" in the category $L$, we obtain the following commutative diagram in $L$ :

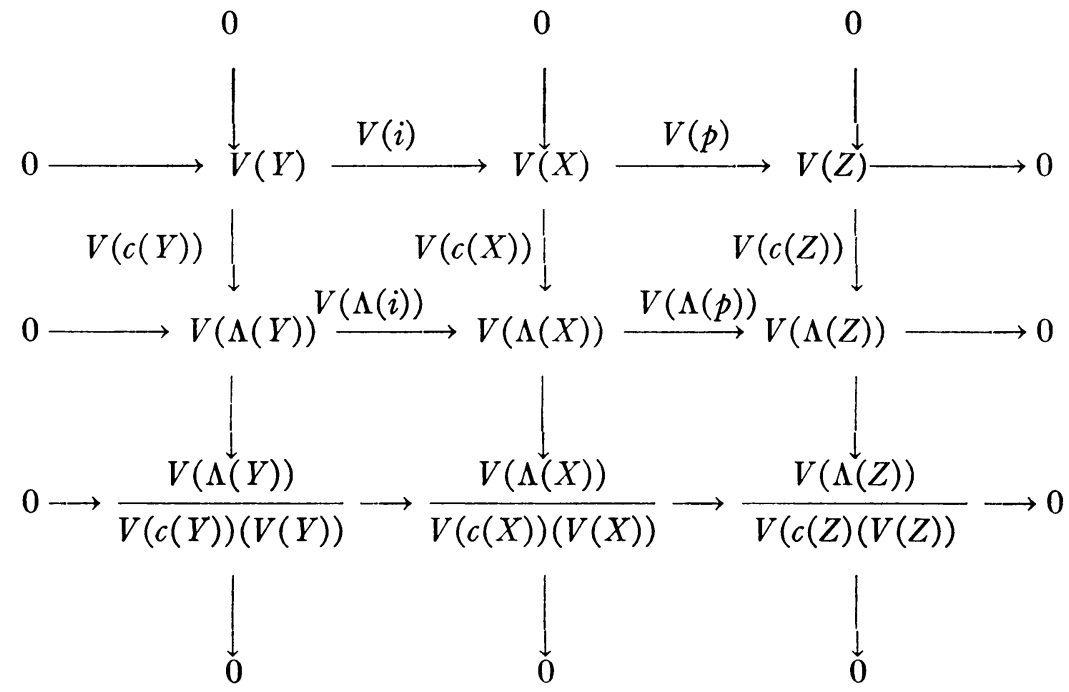

where all columns and rows are exact. Now, observe that $X$ is complete if and only if $V(\Lambda(X)) / V(c(X))(V(X))=0$. The proposition follows immediately.

Clearly, we have not used the full power of our main theorem. In a subsequent paper a number of more interesting applications of this theorem shall be studied.

Finally, the author wishes to express his thanks to the referee for greatly improving the style of presentation of this paper.

Western Michigan University 